\title{
Psychiatric aspects of chronic exposure to organophosphates: diagnosis and management
}

\author{
Robert Davies, Ghouse Ahmed \& Tegwedd Freer
}

In a recent article (Davies et al, 2000) we covered the background to and clinical presentation of the neuropsychiatric consequences of chronic exposure to organophosphates. In particular, we dicussed a syndrome that appears to develop in about $10 \%$ of chronic exposees: chronic organophosphate-induced neuropsychiatric disorder (COPIND). In this article we consider the diagnosis and management of patients with this condition, and related issues.

\section{Diagnosis}

The diagnosis of COPIND is essentially clinical, and Ahmed \& Davies (1997) propose operational diagnostic criteria (Box 1). Failure to meet these criteria does not necessarily exclude a diagnosis of COPIND, but it suggests that other diagnoses should be more vigorously explored.

\section{Assessment}

If possible, in addition to the patient, a close relative or friend should be interviewed. Many patients are reluctant to disclose sensitive information (for example, about suicidal thinking or behaviour), and a close informant may be able to shed valuable light on such matters or to prompt the patient to recall poorly remembered events.

For clinical purposes, a joint interview is probably most helpful, but for medico-legal reports, separate

\section{Box 1 Diagnostic criteria for COPIND}

1 Repeated exposure to organophosphates

2 At least four of the following:

(a) personality change and destabilisation of mood

(b) impairment of concentration and memory

(c) impaired exercise tolerance

(d) reduced tolerance to alcohol

(e) heightened sensitivity to organophosphates.

3 At least three of the following:

(a) exacerbation of 'dippers' flu'

(b) impulsive suicidal thinking

(c) language disorder

(d) heightened sense of smell

(e) deterioration of handwriting

interviews are essential to provide independent corroboration of the patient's account.

The psychiatrist should not be misled by the apparent normality of the patient. The mood swings of COPIND are unpredictable in their timing and duration, and many patients conceal a significant degree of suffering that may be revealed by an informant.

As with any psychiatric interview, open questions should be asked wherever possible. However, it is

Robert Davies is a consultant psychiatrist in Somerset (Rydon House Mental Health Unit, Cheddon Road, Taunton TA2 7AZ; tel.: 01823 333438). Ghouse Ahmed is a locum consultant psychiatrist and has been an Associate Specialist in Somerset since 1972. Tegwedd Freer is a consultant in adult and old age psychiatry at Exeter \& District Community Health Trust. All the authors have been involved in recent years in research into the neuropsychiatric effects of exposure to organophosphates. 
inevitable that some degree of leading will be needed, particularly when enquiring about the key symptoms of COPIND. A detailed history of exposure is generally not required for clinical purposes, for which it is sufficient merely to establish exposure. (Needless to say, a detailed exposure history is mandatory for medico-legal reports.) National Poisons Information Centres can be contacted to determine the nature of any proprietary products. Not all patients will be referred specifically with organophosphate exposure in mind. The symptoms of COPIND may emerge from a more general psychiatric consultation, for example, following attempted suicide. In these cases, systematic enquiry should be made about other symptoms of the syndrome and about possible sources of organophosphate exposure, unsuspected by the patient.

\section{Special investigations}

There are at present no investigations that may be used to establish a clinical diagnosis of COPIND. Blood cholinesterase levels are invaluable in dealing with acute organophosphate exposure, but as they normalise within 10-14 days of the last significant exposure they cannot be used in the assessment of the chronically exposed patient.

Tests of peripheral and autonomic nerve function (Jamal, 1997) may establish damage due to chronic exposure, but some patients with COPIND show no such damage, while others with clear peripheral neuropathy due to organophosphates show few, if any, symptoms of COPIND. The overlap, however, is large, and positive neurophysiological findings may add considerable weight to a clinical diagnosis of COPIND in medico-legal cases.

There is anecdotal evidence that psychometric testing may reveal deficits, but considerably more work is required to establish its diagnostic role.

\section{Management}

The most important step, certainly for the patient, is making the diagnosis and acknowledging that the patient's ill health is related to exposure to organophosphates. This, of itself, can be of tremendous therapeutic benefit, since the overwhelming majority of patients will have had a sequence of medical encounters in which their concerns and fears about the role of organophosphates in their illness have been discounted. Many patients find diagnosis is all that is required, and they organise their lives as best they can in the knowledge that
Box 2 Management of COPIND

Establish diagnosis

Explain to the patient (and family members, if present)

Consider low-dose SSRIs for mood disorder Consider problem-solving counselling Consider family interventions

Liaise closely with the general practitioner

they have an accepted disability with an acknowledged cause. In occupational health terms, they have a 'prescribed disease' (an illness that arises in association with work or occupation and is recognised by the UK Industrial Injuries Scheme). For further information on prescirbed diseases see, for example, Harrington \& Gill (1987).

More seriously affected patients, however, require further intervention. There is only anecdotal evidence for the efficacy or otherwise of treatments tried, and therefore what follows is based on our clinical experience.

\section{Antidepressants}

The most distressing psychiatric symptoms for the patient are mood swings and suicidal thinking. We have found that selective serotonin reuptake inhibitors (SSRIs) can be beneficial for both of these, benefits being reported in well under the 10-14 day period normally required for antidepressants to take effect. A significant proportion of patients are exquisitely sensitive to the adverse effects of a variety of psychotropic drugs and may become very ill with standard doses of SSRIs. Accordingly, we recommend the use of SSRIs available in liquid form (paroxetine $2 \mathrm{mg} / \mathrm{ml}$ or fluoxetine $4 \mathrm{mg} / \mathrm{ml}$ ) and their very gradual introduction and dose increment. A typical regime for paroxetine would be to start on $1 \mathrm{ml}$ per day and increase by $1 \mathrm{ml}$ every 2 days until adverse effects are noted and then drop back to the previous dose and maintain. A similar regime for fluoxetine would rationally involve a longer incremental period, in view of its half-life, but this would need to be balanced against the urgency of introducing effective treatment. Typical effective doses in sensitive patients are of the order of 8-10 mg of either drug daily. Many patients, however, can tolerate full therapeutic doses and the drug should be built up to the British National Formulary maximum, tolerance permitting (British Medical Association \& Royal Pharmaceutical Society, 2000). 
We do not recommend the use of tricyclic antidepressants, since a number of patients referred to us who have taken these drugs in the past have developed severe and grossly distressing anticholinergic effects - dry mouth to the point of ulceration, urinary retention in the absence of prostatic disease and severe constipation. This may reflect a combination of down-regulation of acetylcholine receptors and autonomic neuropathy. Others, however, seemed able to tolerate these drugs in moderate dose.

Unfortunately, an unknown proportion of patients derives no, or only transient, benefit from antidepressants.

\section{Psychological treatment}

Cognitive therapy is frequently recommended in chronic fatigue syndrome (CFS), but our experience of patients who have been referred for this disorder is universally one of rejection. The reason for this appears to be that often the cognitive strategy adopted seems aimed at focusing the patients' thoughts away from the causative agent and the belief that they have suffered physical damage as a result of exposure. We would suggest that psychological approaches similar to those used in chronic or degenerative neurological conditions such as multiple sclerosis and motor neuron disease are most appropriate, as they essentially involve problem solving and coming to terms with the disability. Accordingly, our recommendation is for counselling and support, rather than formal psychotherapy, unless there is a clear comorbid condition indicating the latter.

\section{General management}

The prognosis for organophosphate-induced disorder is uncertain. In most cases it appears to run a chronic but stable course. In a proportion, however, a more malignant process seems to be present, with progressive deterioration in the absence of further exposure to organophosphates. We are aware of no cases of recovery.

Emerging evidence of premature osteoporotic change in people exposed to organophosphates (Compston et al, 1999) means that a referral for bone density studies would seem to be an ethical part of any management plan, if these have not already been carried out.

It cannot be emphasised too strongly that organophosphate exposure leads to multi-system disorder. Peripheral and autonomic neuropathy, cardiorespiratory problems, possible premature osteoporosis and the symptoms of COPIND combine to create a degree of suffering in which a close partnership between the general practitioner and the mental health team is essential.

\section{The suicidal patient}

Suicidal thinking in organophosphate victims tends to come on suddenly and abate quickly. In general, it is unlikely that a patient will be actively suicidal when he or she sees a psychiatrist or other mental health professional. However, this should not lead to a false sense of security, as further episodes are likely to occur.

It is probable that, at this stage of recognition of the problem, there is little that psychiatric services alone can do to prevent suicides. Anecdotally, the most effective preventive measure seems to be the establishment of local informal self-help groups, where suicidal patients can be talked down by others who have had the same experience. Generic helplines are perceived (again anecdotally) as unhelpful, as they tend to focus on factors other than the illness.

Where active suicidal thinking is detected, the patient should be admitted to hospital and closely observed: the risks are very high, particularly if comorbid major affective disorder is present.

While the typical picture is of brief, impulsive suicidal thinking, a proportion of exposees, as a result of their debilitating condition, may become more persistently suicidal. In the absence of clinical depression, exposees suffer emotional and physical problems similar to those experienced by people in chronic, intractable pain. In such cases, close support is indicated, but it is still possible that the patient will attempt or commit 'rational suicide', in view of the poor prognosis of the condition.

\section{Management of the family}

The effects of organophosphate toxicity affect not only the patient, but also the family. Frequently, the ability to earn a living is lost, dramatically reducing the family's financial circumstances. Mood swings, in particular, may have a major and adverse effect on the family, especially the children. One of us (R.D.) had the poignant experience of hearing a 5-year-old tell how she insisted on "daddy taking his medicine because if he didn't he would become nasty".

It is to be hoped that increasing recognition of the reality and seriousness of COPIND will stimulate development of models of family intervention. The 
psychiatrist may be placed in an advocacy role, particularly when children develop problems. It should be borne in mind that children (particularly those of agricultural exposees) may themselves have been exposed and suffered damage. Reports of behavioural oddities, poor school performance and unusual physical symptoms should therefore raise the possibility of a direct toxic effect. Such children should not be forced into conventionally accepted diagnostic categories, although, of course, a significant proportion of their problems may be related to family dynamics disturbed as a result of COPIND, rather than to direct toxicity. This is an area that requires urgent collaborative research by child and adolescent psychiatrists and paediatricians.

\section{Comorbidity}

The development of COPIND does not preclude the possibility that patients will also have more conventional psychiatric disorders. In general, the management of these should be as for the primary illness, but with the caveat that patients may be exceptionally sensitive to psychotropic drugs, which must therefore be introduced very cautiously. The case report and aetiological hypothesis published by Bradwell (1994) merit close consideration.

Electroconvulsive therapy (ECT) raises particular concerns. Significant anaesthetic complications arising from the use of muscle relaxants have been reported (e.g. Jaksa \& Palahniuk, 1995). Although these have generally been in the context of recent exposure, given the effects of organophosphates on neuromuscular function (Jamal, 1997) great caution must be exercised. Accordingly, if ECT is clinically indicated and there is evidence of organophosphate damage or recent exposure, it should be administered in conditions where intensive life support systems are available. Under no circumstances should it be given in units with facilities less than those of a district general hospital.

\section{Medico-legal factors}

The most obvious area of medico-legal activity is personal injury litigation. Compensation for damage due to chronic exposure has been sought in only a few cases, all of which have been won or settled advantageously out of court, for sums ranging from the modest to the substantial. It is likely, therefore, that the psychiatrist dealing with a patient with COPIND will at some stage be asked to provide a medical report. Experience of such litigation leads us to warn psychiatrists of the unusually acrimonious nature of these cases, in which the defence and its experts go to considerable lengths to attempt to demolish the credibility of the plaintiff's expert. The stakes are high for the defendants. The psychiatrist, therefore, should take on such cases only if fully confident of the facts and his or her expertise in this area, and should have extensive conferences with Counsel before taking the stand.

Swings of mood into irritability and anger are an intrinsic part of COPIND, and in a small number of cases they may result in overt aggression, violence and criminal proceedings. Offenders with the features of COPIND may increasingly seek mitigation for offences of violence. There is anecdotal evidence of extreme acts of violence, without antecedents and out of character, committed in the context of organophosphate exposure (Devinsky et al, 1992). Therefore the psychiatrist examining a violent offender who acts out of character and who has a significant history of organophosphate exposure should consider the possibility that this may play a part in the offending behaviour. The question of mens rea (criminal intent) and its absence (for example, owing to the interaction between alcohol, COPIND and recent organophosphate exposure) is beyond the scope of this paper.

\section{Organophosphates and Gulf War illness}

The Royal Colleges of Physicians' and Psychiatrists' joint report (1998) referred to the problems encountered by those exposed to organophosphate sheep dips. UK service personnel involved in Desert Shield and Desert Storm deployed in the Gulf were regularly and heavily exposed to organophosphate pesticides, including diazinon, which is probably the most popular sheep dip. This fact was admitted by Sir Christopher Soames, then Secretary of State for Defence. French troops deployed in the Gulf, however, used synthetic pyrethroids for insecticidal and other pesticidal purposes, and the French military have reported no cases of anything resembling Gulf War illness. Troops from the USA, however, like their UK counterparts, were exposed to organophosphates and they also report relatively high rates of ill health involving significant psychiatric symptomatology.

Government-funded research conducted in the UK (Ismail et al, 1999; Unwin et al, 1999) found a significantly increased level of ill health in Gulf War veterans compared with service personnel deployed 
in Bosnia, but failed to identify any significant and distinctive pattern of illness. Independently funded researchers in the USA (Haley \& Kurt, 1997; Haley et al, 1997a, b), however, produced compelling evidence of neuropsychiatric disorder in US Gulf War veterans, which they linked with both pesticide use and possible exposure to organophosphate military nerve agents following the destruction of the Iraqi munitions depot at Khammisiya. It is beyond the scope of this paper analyse these research projects in detail. We would note, however, that the UK group's questionnaire would not have detected COPIND, as it asked about only two of the ten symptoms required and did not distinguish between organophosphates and other pesticides.

From a clinical standpoint, the few Gulf War veterans that we have seen are largely indistinguishable, within the terms of COPIND, from sheep farmers and others exposed to organophosphates. However, they seem to display a wider variety of non-psychiatric symptoms, some extremely disabling, for which there are many potential explanations. Our advice to psychiatrists in civilian practice asked to deal with Gulf War veterans who present with emotional and cognitive problems is seriously to consider the involvement of organophosphate exposure and to enquire about the symptoms of COPIND that may not be readily volunteered, particularly impulsive suicidal thinking. If a match is found then we would recommend measures similar to those we advocate for agricultural and other civilian organophosphate exposees.

\section{Post-traumatic stress disorder and Gulf War illness}

Given the circumstances of exposure and the experiences that some Gulf War personnel endured, post-traumatic stress disorder (PTSD) remains an important differential diagnosis. However, in dealing with such patients, it is critical to remember that they are highly trained and experienced professionals, prepared for the risks and dangers of military life. If their symptoms closely resemble those of sheep dippers and others exposed to organophosphates, attribution of these to PTSD should be made with circumspection.

Nevertheless, there will be those with comorbidity and they would seem to be at very high risk of suicide and aggressive behaviour, possibly to the point of homicide. Such individuals must be recognised and intensively engaged by informed professionals. It is hoped that those expert in the management of PTSD will take due note of the risk-elevating effect of chronic organophosphate exposure.

\section{Summary}

This is a brief overview of a highly contentious subject. Many maintain that chronic 'low-dose' exposure to organophosphate pesticides is safe and does not cause physical or mental ill health. Increasingly, those who take this view are losing ground. The relationship between organophosphates and CFS is complex. Our view is that organophosphates induce a clinical state that is essentially different from CFS, with significant implications for management, particularly if specific anticholinesterases are advocated as treatment. A comparison between symptom rates in COPIND (Davies et al, 1999) and in CFS (Komaroff, 1993) is recommended.

We are very cautiously optimistic that a combination of appropriately titrated antidepressant medication and counselling, augmented where necessary by family interventions, can do much to lessen the great suffering of patients chronically exposed to organophosphates. Without doubt, however, the most positive therapeutic intervention available at present is the recognition of their condition and its cause, which relieves them of much self-doubt and (now decreasing) opprobrium.

For the psychiatrist, the key message is that organophosphate damage exists in a wide variety of individuals (not just sheep dippers). We therefore recommend that when symptoms of affective instability coupled with subjective cognitive impairment and either fatigue or inability to maintain muscular activity are encountered, systematic enquiries should be made concerning organophosphate exposure and the other symptoms of COPIND before a treatment plan is formulated. The multi-system nature of the disorder means that treatment plans need to be holistic. Thus, while psychiatrists and other mental health professionals have a major role to play, only the patient's general practitioner is in a position fully to coordinate management.

\section{References}

Ahmed, G. M. \& Davies, D. R. (1997) Chronic organophosphate exposure: toward the definition of a neuropsychiatric syndrome. Journal of Nutritional and Environmental Medicine, 7, 169-176.

Bradwell, R. H. (1994) Psychiatric sequelae of organophosphate poisoning: a case study and review of the literature. Behavioural Neurology, 7, 117-122.

British Medical Association \& Royal Pharmaceutical Society (2000) British National Formulary. London \& Wallingford: BMJ Books \& Pharmaceutical Press. 
Compston, J. E., Vedi, S., Stephen, A. B. et al (1999) Reduced bone formation after exposure to organophosphates. Lancet, 354, 1791-1792.

Davies, D. R., Ahmed, G. M. \& Freer, T. (1999) Chronic organophosphate induced neuropsychiatric disorder (COPIND): results of two postal questionnaire surveys. Journal of Nutritional and Environmental Medicine, 9, 123-134.

— _ _ \& - (2000) Psychiatric aspects of chronic exposure to organophosphates: background and clinical picture. Advances in Psychiatric Treatment, 6, 187-192.

Devinsky, O., Kernan, J. \& Bear, D. M. (1992) Aggressive behaviour following exposure to cholinesterase inhibitors. Journal of Neuropsychiatry and Clinical Neuroscience, 4, 189-194.

Haley, R. W. \& Kurt, T. L. (1997) Self reported exposure to neurotoxic chemical combinations in the Gulf War. Journal of American Medical Association, 277, 231-237.

— _ _ \& Horn, J. (1997a) Is there a Gulf War Syndrome? Journal of American Medical Association, 277, 215-222.

-, Horn, J., Roland, P. S., et al (1997b) Evaluation of neurological function in Gulf War veterans. Journal of American Medical Association, 277, 223-230.

Harrington, J. M. \& Gill, F. S. (1987) Occupational Health. Oxford: Blackwell.

Ismail, K., Everett, B., Blatchley, N., et al (1999) Is there a Gulf war syndrome? Lancet, 353, 179-182.

Jaksa, R. J. \& Palahniuk, M. J. (1995) Attempted organophosphate suicide: a unique cause of prolonged paralysis during electroconvulsive therapy. Anesthesia and Analgesia, $80,823-823$.

Jamal, G. A. (1997) Neurological syndromes of organophosphorus compounds. Adverse Drug Reactions and Toxicology Reviews, 16, 133-170.

Komaroff, A. L. (1993) Clinical presentation of chronic fatigue syndrome. In CIBA Foundation Symposium 173 (eds G. R. Bock \& J. Whelan), pp. 43-61. Chichester. John Wiley \& Sons.

Royal College of Physicians \& Royal College of Psychiatrists (1998) Organophosphate Sheep Dip. Clinical Aspects of LongTerm Low-Dose Exposure. Report of a Joint Working Party of the Royal College of Physicians and Royal College of Psychiatrists. Council Report CR67. London: Royal College of Physicians.

Unwin, C., Blatchley, N., Coker, W., et al (1999) Health of UK servicemen who served in the Persian Gulf. Lancet, 353, 169-178.

\section{Multiple choice questions}

\section{Patients with COPIND:}

a may not realise they have been exposed to organophosphates

b always show evidence of peripheral neuropathy

c may be Gulf War veterans

d should be routinely treated with tricyclic antidepressants

e respond well to cognitive therapy.
2. The families of patients with COPIND:

a may make useful contributions to the assessment of the patient

b should never be interviewed with the patient present

c may require psychiatric intervention

d may also suffer from organophosphate damage

e may suffer financially as a result of the patient's illness.

3. In dealing with COPIND, the psychiatrist:

a should not reveal the diagnosis to the patient

b may have to act as an advocate for other family members

c should consider alternative diagnoses

$\mathrm{d}$ may be called as an expert witness in court

e is ideally placed to be the case coordinator.

\section{MCQ answers}

$\begin{array}{lllll}\text { 1 } & \text { 2 } & \text { 3 } & \\ \text { a } & \text { T } & \text { a T } & \text { a } & \text { F } \\ \text { b F } & \text { b F } & \text { b } & \text { T } \\ \text { c T } & \text { c T } & \text { c } & \text { T } \\ \text { d F } & \text { d T } & \text { d } & \text { T } \\ \text { e F } & \text { e T } & \text { e } & \text { F }\end{array}$

